\title{
Socioeconomic and clinical factors affecting the proportion of breast conserving surgery in Chinese women with breast cancer
}

\author{
Jingjing Liu ${ }^{1,2,3,4,5}$, Dongdong Zhou ${ }^{1,2,3,4,5}$, Teng Pan ${ }^{1,2,3,4,5}$, Lixia Cao ${ }^{1,2,3,4,5}$, Qianxi Yang ${ }^{1,2,3,4,5}$, Jin Zhang ${ }^{1,2,3,4,5}$ \\ ${ }^{1}$ 3rd Department of Breast Cancer, China Tianjin Breast Cancer Prevention, Treatment and Research Center, Tianjin Medical University Cancer \\ Institute and Hospital, Tianjin, China; ${ }^{2}$ Key Laboratory of Breast Cancer Prevention and Therapy of Ministry of Education; Tianjin, China; ${ }^{3}$ Key \\ Laboratory of Cancer Prevention and Therapy, Tianjin, China; ${ }^{4}$ Tianjin's Clinical Research Center for Cancer, Tianjin, China; ${ }^{5}$ Tianjin Medical \\ University, Ministry of Education, National Clinical Research Center for Cancer, Tianjin, China \\ Contributions: (I) Conception and design: J Liu; (II) Administrative support: J Zhang; (III) Provision of study materials or patients: J Liu, D Zhou; (IV) \\ Collection and assembly of data: T Pan, L Cao; (V) Data analysis and interpretation: D Zhou, Q Yang; (VI) Manuscript writing: All authors; (VII) \\ Final approval of manuscript: All authors. \\ Correspondence to: Jin Zhang. Tianjin Medical University Cancer Institute and Hospital, Huanhuxi Road, He Xi District, Tianjin 300060, China. \\ Email: zhangjintjmuch1@163.com.
}

Background This study investigated the socioeconomic and clinical factors affecting the proportion of breast conserving surgery (BCS) in China, to improve the proportion and success rate of BCS in Chinese breast cancer patients.

Methods: Six hundred and forty breast cancer patients treated with BCS were compared with 700 selected breast cancer patients (controls) treated with modified radical mastectomy (MRM) in Tianjin Medical University Cancer Institute and Hospital from January 2005 to January 2018. Patients' socioeconomic and clinical factors were collected through telephone interviews or face-to-face interviews. A total of 5,660 BCS patients were enrolled to analyze independent factors affecting initial positive margins. Chi-squared test and multiple logistic regressions were used to examine factors associated with BCS. The locoregional recurrence-free survival (LRRFS), distant metastasis-free survival (DMFS), and overall survival (OS) were calculated using the KaplanMeier method and the survival distribution between BCS and MRM groups was compared by log-rank test.

Results: Breast cancer patients who were younger, lived in urban areas, had medical insurance, and higher levels of education and Personal income were more likely to choose BCS. We also observed that patients of Han nationality were more likely to choose BCS. Univariate analysis showed that the frozen section analysis (FSA) positive margin was significantly correlated with tumor distance from the nipple, preoperative magnetic resonance imaging (MRI) examination, T stage, pathological subtype, and lymphovascular invasion (LVI). Multivariate analysis showed the distance from the nipple, T stage, pathological subtype, and LVI, and no preoperative MRI examination were independent predictors of positive resection margins. Multivariate analysis of the correlation between MRI findings and positive resection margins revealed that tumor size, non-mass enhancement (NME), and malignant enhancement surrounding the tumor were independent predictors of positive resection margins.

Conclusions: In China, socioeconomic factors largely influence the choice of surgical procedures for breast cancer patients. A gradual reduction in the influence of socioeconomic factors on the proportion of BCS is recommended. Furthermore, preoperative MRI should be encouraged in patients preparing for BCS. Clinicopathological characteristics and MRI findings are significantly associated with a positive resection margin in breast cancer patients.

Keywords! Breast conserving surgery (BCS); socioeconomic status; margin; magnetic resonance imaging (MRI)

Submitted Dec 05, 2021. Accepted for publication Feb 16, 2022.

doi: 10.21037 /gs-22-25

View this article at: https://dx.doi.org/10.21037/gs-22-25 


\section{Introduction}

The National Surgical Adjuvant Breast and Bowel Project (NSABP) and European Milan trial confirmed that breast conserving surgery (BCS) in combination with radiotherapy showed no significant difference in diseasefree survival (DFS) and overall survival (OS) compared with mastectomy $(1,2)$. BCS has become the standard treatment for early breast cancer, and compared with mastectomy, is associated with reduced psychological burden, better cosmetic outcomes, and a reduced risk of wound infection $(3,4)$. This has led to an increase of BCS in many countries. A report from Canada showed that the breast conserving rate has been on the rise since the 1970s. In the United States, Surveillance, Epidemiology, and End Results (SEER) data showed this was 55-60\% between 2000 and 2006 (4), while In Europe, data from more than 20 breast cancer treatment centers certified by the European Society of Breast Cancer Specialists (EUSOMA) from 2003 to 2010 showed that the rate was between $75 \%$ and $80 \%$ (5). In China, since the 1990 s, BCS has gradually become the recommended surgical treatment for early breast cancer (6), and even in developed regions such as Beijing and Shanghai, the rate of BCS rose from $12.1 \%$ in 2005 to $24.3 \%$ in 2008 . Between 2005 and 2018, only 16.8\% of 51,678 breast cancer patients receiving surgical treatment in our hospital chose BCS (6). However, in the past 3 years, this has risen to about $24.7 \%$. Therefore, it is necessary to study the reasons for the low proportion of BCS in China. Retrospective study had found that breast cancer patients' socioeconomic status is the predominant factor in determining whether a breast cancer patient receives breast conserving therapy (BCT) or not in China in 2012 (7). Are there other socio-economic factors affecting the implementation of BCS in the past decade? And doctors should also pay attention to the clinical factors that improve the success rate of BCS in the process of clinical treatment on the other hand. Therefore, it is necessary to study the socioeconomic and clinical factors reasons for the low proportion of BCS in China.

The first objective of the current study was to compare the socioeconomic status of BCS and modified radical mastectomy (MRM) patients in order to understand what prevents eligible breast cancer patients from receiving BCS. Not all women with breast cancer are appropriate candidates for BCT. Patient with T stage 1-2; and negative intraoperative frozen section analysis (FSA) of the margin. Most breast cancer treatment guidelines state that the expected cosmetic outcome and patient preferences should guide the decision to perform BCS. We hypothesized on the premise of meeting the requirements of BCS, patients with higher level of education, lived in urban areas, nationality, had medical insurance and personal income are the factors that may influence the patients' options in receiving BCT.

The most important disadvantage of BCS is the possibility of tumor at or near the excised edge (8). Therefore, BCS needs to remove the tumor and ensure there are no residual tumor cells at the surgical margin, to reduce the risk of local recurrence. Positive section margins found in the final paraffin sections of some patients require reoperation, which leads to a delay in the initiation of adjuvant therapy, an increase in the amount of mastectomy compared to the primary surgery, poor appearance, increased anesthesia risk, patient anxiety, higher cost, and an increased recurrence rate (9). Therefore, it is essential to analyze the influencing factors of a positive resection margin of the FSA and how to improve the primary success rate of BCS. Breast magnetic resonance imaging (MRI) has been widely used in breast cancer patients to assess tumor status, and previous studies reported it could improve the success rate of BCS $(10,11)$. Therefore, it is important to analyze preoperative MRI findings associations with a positive resection margin during BCS.

We present the following article in accordance with the STROBE reporting checklist (available at https:// gs.amegroups.com/article/view/10.21037/gs-22-25/rc).

\section{Methods}

\section{Socioecnomic features}

\section{Patients and materials}

A total of 51,237 breast cancer patients were treated in the Tianjin Medical University Cancer Institute and Hospital from January 2005 to January 2018, among whom 8,640 patients underwent BCS. We randomly selected 640 breast cancer patients (stage I or II) treated with BCS from January 2005 to January 2018 according to their admission number and compared them with 700 breast cancer patients who met the criteria for BCS but were treated with MRM during the same time period. All patients were pathologically confirmed to have primary breast cancer. Patient information was collected through telephone interviews or face-to-face interviews and included age, residence, medical insurance, Personal income, education level, and nationality.

All procedures performed in this study were in 
accordance with the Declaration of Helsinki (as revised in 2013). The study was approved by the Ethics Committee of Tianjin Medical University Cancer Institute and Hospital (No. bc2015014) and the need for individual consent was waived due to the retrospective nature of the present study.

\section{Clinical and pathological features}

\section{Patients and materials}

The clinical and pathological data of 5,660 breast cancer patients who had received intraoperative FSA of the margin from 2005 to 2018 in the Tianjin Medical University Cancer Institute and Hospital was collected.

Patient inclusion criteria were (I) T stage 1-2, and (II) FSA of the margin. Patient exclusion criteria were (I) neoadjuvant chemotherapy was performed before surgery and (II) previous history of breast cancer. Among the 5,660 patients 3,169 had preoperative MRI. MRI findings were collected to analyze their association with a positive margin.

\section{Surgical procedure and pathology}

During the BCS, the macroscopically visible margins of normal tissue about $2 \mathrm{~cm}$ around the tumor were removed to ensure sufficient negative margins. After removal of the tumor-containing specimens, frozen sections about $0.5 \mathrm{~cm}$ thick around the resected specimens were made, and the corresponding margin positions were marked for pathological evaluation. If the result of the FSA was negative, the BCS was considered successful, and if the result was positive, further resection or mastectomy was chosen according to the judgment of the operator.

\section{MRI technique and analysis}

MRI technology: breast MRI was performed by a $3.0 \mathrm{~T}$ superconducting whole-body MRI scanner (GE Company, Boston, MA, USA). The patient was in the prone position with the breast hanging in the coil and the chest wall close to the coil. Conventional plain scanning was performed first, and the imaging sequence was axial lipid pressure T2weighted imaging (T2WI), followed by dynamic enhanced scanning. A high-pressure syringe was used to inject the $15 \mathrm{~mL}$ of the contrast agent gadolinium dexamethasone at a speed of $2.5 \mathrm{~mL} / \mathrm{s}, 15$ and $20 \mathrm{~mL}$ normal saline was applied to flush at the same speed. Image analysis was conducted by two associate chief physicians with more than 5 years of experience in breast MRI diagnosis. The tumor location, size, enhancement shape, and dynamic enhancement were determined mainly based on lipid pressure T2WI, diffusion- weighted imaging (DWI), and dynamic enhancement images curve according to the American Radiology Breast Imaging and Reporting System (BI-RADS) in qualitative diagnosis of breast lesions.

\section{Statistical methods}

Initial descriptive statistics included comparisons between BCS cases and MRM controls for these characteristics using chi-square test. Multivariate analysis was performed using logistic regression analysis of statistically significant variables $(\mathrm{P}<0.05)$ via univariate analysis to assess factors associated with BCS. Receiver operating characteristic (ROC) curve analysis was performed to assess distance from the nipple, The following variables were included in the logistic regression analyses: age, residence, educational background, personal income, medical insurance and Nationality. All statistical tests were performed using SPSS 20.0 software (IBM Corporation, Armonk, NY, USA) and results were considered significant at a $\mathrm{P}$ value $<0.05$.

\section{Survival analysis}

Locoregional recurrence-free survival (LRRFS) was defined as the period from the date of the disease diagnosed to the date when local recurrence occurred. Distant metastasis-free survival (DMFS) was defined as the period from the date of the disease diagnosed to the date when distant recurrence occurred. The OS was defined as the time from the date of diagnosis to the date of death or loss of the follow-up. All follow-ups ended on December 31, 2017. The LRRFS, DMFS, and OS were calculated using the Kaplan-Meier method and the survival distribution between groups was compared by log-rank test. All data were collected completely.

\section{Results}

\section{Socioeconomic features}

A total of 1,340 breast cancer patients were included in this study, and comparison of socioeconomic factors between BCS and MRM patients are shown in Table 1. Compared with the MRM patients, BCS patients were significantly younger, lived in urban areas, were of the Han nationality, had medical insurance, had higher personal income, and were well educated (Table 1).

Multivariate analysis of BCS socioeconomic influencing factors showed breast cancer patients who were younger, 
Table 1 Comparison of socioeconomic influencing factors between MRM and BCS patients

\begin{tabular}{|c|c|c|c|c|}
\hline Factors & BCS & MRM & $\chi^{2}$ & $P$ \\
\hline Age (years) & & & 173.533 & $<0.01$ \\
\hline$<40$ & 215 & 60 & & \\
\hline $40-60$ & 320 & 581 & & \\
\hline$>60$ & 105 & 59 & & \\
\hline Residence & & & 14.653 & $<0.01$ \\
\hline Urban & 448 & 420 & & \\
\hline Rural & 192 & 280 & & \\
\hline Medical insurance & & & 51.331 & $<0.01$ \\
\hline Yes & 576 & 525 & & \\
\hline No & 64 & 175 & & \\
\hline Personal income (yuan) & & & 44.081 & $<0.01$ \\
\hline$<1,000$ & 13 & 77 & & \\
\hline $1,000-3,000$ & 448 & 462 & & \\
\hline$>3,000$ & 179 & 161 & & \\
\hline Educational background & & & 31.918 & $<0.01$ \\
\hline Primary school & 57 & 122 & & \\
\hline Junior middle school & 435 & 380 & & \\
\hline Senior high school and above & 148 & 198 & & \\
\hline Nationality & & & 5.761 & 0.016 \\
\hline Han nationality & 608 & 642 & & \\
\hline Minority nationality & 32 & 58 & & \\
\hline
\end{tabular}

MRM, modified radical mastectomy; BCS, breast conserving surgery.

lived in urban areas, had medical insurance, had higher levels of education and a high Personal income were more likely to choose BCS. We also observed that the Han nationality were more likely to choose BCS [odds ratio (OR) $=1.817 ; \mathrm{P}=0.018]$ (Table 2).

\section{Clinical and pathological features}

Clinicopathological factors and positive section margin A total of 5,660 breast cancer patients who had planned to undergo BCS and were analyzed by intraoperative frozen section of the incision margin were included, as shown in Table 3. The clinicopathological data of the patients and the univariate analysis results of the positive margin influencing
Table 2 Multivariate analysis of BCS socioeconomic influencing factors

\begin{tabular}{|c|c|c|c|}
\hline Factors & OR & $95 \% \mathrm{Cl}$ & $\mathrm{P}$ \\
\hline \multicolumn{4}{|l|}{ Age (years) } \\
\hline$<40$ & 2.785 & $1.756-4.417$ & $<0.001$ \\
\hline $40-60$ & 0.312 & $0.215-0.454$ & $<0.001$ \\
\hline$>60$ & Ref & & \\
\hline \multicolumn{4}{|l|}{ Residence } \\
\hline Urban & 2.247 & $1.722-2.933$ & $<0.001$ \\
\hline Rural & Ref & & \\
\hline \multicolumn{4}{|l|}{ Personal income (yuan) } \\
\hline$<1,000$ & 0.257 & $0.124-0.535$ & 0.001 \\
\hline $1,000-3,000$ & 0.976 & $0.738-1.291$ & 0.865 \\
\hline$>3,000$ & Ref & & \\
\hline \multicolumn{4}{|l|}{ Educational background } \\
\hline Primary school & 0.401 & $0.271-0.581$ & $<0.001$ \\
\hline Junior middle school & 1.313 & $0.853-2.020$ & 0.216 \\
\hline Senior high school and above & Ref & & \\
\hline \multicolumn{4}{|l|}{ Nationality } \\
\hline Han nationality & 1.817 & $1.110-2.974$ & 0.018 \\
\hline Minority nationality & Ref & & \\
\hline
\end{tabular}

factors of the initial surgical margin are shown in Table 4 .

Table 3 shows the overall success rate of BCS was $83.6 \%$, and $14.6 \%$ of patients obtained a negative margin by expanded resection which was treated with BCS. Only $1.1 \%$ of patients could not reach the negative margin state after resection, and finally underwent mastectomy. However, $15.3 \%$ of patients who were initially positive for the intraoperative frozen resection margin were chosen for mastectomy.

The goal of BCS is to completely remove the tumor and achieve a negative margin while providing satisfactory cosmetic results. Therefore, this study further analyzed the influencing factors of an initial positive resection margin, and the clinicopathological characteristics and univariate analysis results are shown in Table 4. This reveals a FSA initial positive resection margin is significantly correlated with the distance between the tumor and the nipple $(\mathrm{P}=0.008)$, $\mathrm{T}$ stage $(\mathrm{P}<0.001)$, pathological type $(\mathrm{P}<0.001)$, 
Table 3 Surgical methods

\begin{tabular}{lccc}
\hline Surgical procedures & Number of case & Effective percentage (\%) & Cumulative percentage (\%) \\
\hline Initial negative margin & 3,906 & 69.0 & 69.0 \\
Extended resection negative margin & 826 & 14.6 & 83.6 \\
Extended resection positive margin & 62 & 1.1 & 84.7 \\
Mastectomy after initial positive margin & 866 & 15.3 & 100.0 \\
Total & 5,660 & 100.0 & - \\
\hline
\end{tabular}

Table 4 Univariate analysis of factors affecting the initial positive $(n=5,660)$

\begin{tabular}{|c|c|c|c|}
\hline Clinicopathological factors & Positive resection margin $(n=1,754), n(\%)$ & Negative resection margin $(n=3,906), n(\%)$ & $P$ \\
\hline Menopausal status & & & 0.205 \\
\hline Premenopausal & $943(16.7)$ & $2,028(35.8)$ & \\
\hline Postmenopausal & $811(14.3)$ & $1,878(33.2)$ & \\
\hline No & $1,251(22.1)$ & $2,700(47.7)$ & \\
\hline Yes & $503(8.9)$ & $1,206(21.3)$ & \\
\hline Tumor location & & & 0.121 \\
\hline Outer upper quadrant & $910(16.1)$ & $2,150(38.0)$ & \\
\hline Inner upper quadrant & $525(9.3)$ & $934(16.5)$ & \\
\hline Nipple rear & $13(0.2)$ & $81(1.3)$ & \\
\hline Preoperative MRI examination & & & 0.015 \\
\hline No & $814(14.4)$ & $1,677(29.6)$ & \\
\hline Yes & $940(16.6)$ & $2,229(39.4)$ & \\
\hline Pathological type & & & $<0.001$ \\
\hline Non-infiltrating cancer & $345(6.1)$ & $436(7.7)$ & \\
\hline Infiltrating specific cancer & $119(2.1)$ & $210(3.7)$ & \\
\hline T stage & & & $<0.001$ \\
\hline T1 & 1,392 (24.6) & $3,583(63.3)$ & \\
\hline $\mathrm{T} 2$ & $362(6.4)$ & $323(5.7)$ & \\
\hline
\end{tabular}

Table 4 (continued) 
Table 4 (continued)

\begin{tabular}{lcc}
\hline Clinicopathological factors & Positive resection margin $(n=1,754), n(\%)$ & Negative resection margin $(n=3,906), n(\%)$ \\
\hline LVI & $1,307(23.1)$ & $3,571(63.1)$ \\
No & $447(7.9)$ & $335(5.9)$ \\
Yes & & $<0.001$ \\
Molecular subtype & $640(11.3)$ & $1,356(24.0)$ \\
Luminal A & $850(15.0)$ & $1,914(33.8)$ \\
Luminal B & $164(2.9)$ & $352(6.2)$ \\
HER2-enriched & $100(1.8)$ & $284(5.0)$ \\
TNBC
\end{tabular}

MRI, magnetic resonance imaging; LVI, lymphovascular invasion; HER2, human epidermal growth factor receptor-2; TNBC, triple-negative breast cancer.

Table 5 Multivariate analysis of clinicopathological factors affecting initial positive margin

\begin{tabular}{lccc}
\hline Variables & OR & $95 \% \mathrm{Cl}$ & $\mathrm{P}$ \\
\hline Preoperative MRI examination & & & \\
Yes & Ref & & \\
No & 1.291 & $1.110-1.502$ & 0.001 \\
T stage & & & \\
T1 & Ref & & \\
T2 & 2.599 & $2.093-3.228$ & $<0.001$ \\
Pathological type & & & \\
Infiltrating non-specific cancer & Ref & & \\
Non-infiltrating cancer & 26.889 & $22.910-31.561$ & $<0.001$ \\
Infiltrating specific cancer & 4.923 & $3.796-6.386$ & $<0.001$ \\
LVI & & & \\
Yes & Ref & & \\
No & 0.299 & $0.244-0.367$ & $<0.001$ \\
Tumor distance from nipple & 0.830 & $0.700-0.982$ & 0.030 \\
\hline
\end{tabular}

OR, odds ratio; $\mathrm{Cl}$, confidence interval; $\mathrm{MRI}$, magnetic resonance imaging; LVI, lymphovascular invasion.

and lymphovascular invasion $(\mathrm{LVI})(\mathrm{P}<0.001)$, and preoperative MRI examination $(\mathrm{P}=0.015)$.

Multivariate logistic regression analysis showed the distance from the nipple $(\mathrm{OR}=0.830 ; \mathrm{P}=0.03)$, noninfiltrating cancer $(\mathrm{OR}=26.899 ; \mathrm{P}<0.001)$, infiltrating specific cancer $(\mathrm{OR}=4.923 ; \mathrm{P}<0.001)$, $\mathrm{T}$ stage $(\mathrm{OR}=2.599$; $\mathrm{P}<0.001)$, LVI $(\mathrm{OR}=0.299 ; \mathrm{P}<0.001)$, and preoperative

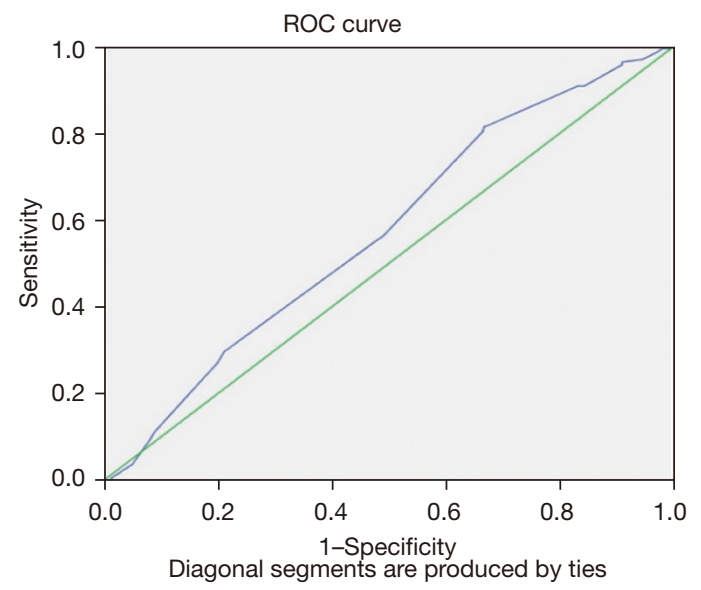

Figure 1 ROC curve analysis of distance from the nipple. ROC, receiver operating characteristic.

MRI examination $(\mathrm{OR}=1.291 ; \mathrm{P}=0.001)$ were independent factors (in Table 5) for FSA initial positive resection margin. In addition, as the closer the tumor was to the nipple, the higher the risk of a positive margin $[\mathrm{OR}=0.830$; 95\% confidence interval (CI): 0.700-0.982; $\mathrm{P}=0.030$ ], we performed ROC curve analysis, and as shown in Figure 1, the area under the curve was 0.572 , with a statistically significant difference compared with $0.5(\mathrm{P}=0.009)$. When the boundary value was $4.55 \mathrm{~cm}$, the difference between the corresponding sensitivity and (1-specificity) was the largest. Tumor size was then divided into two groups with $4.55 \mathrm{~cm}$ as the cut-off value for comparative analysis. The median tumor size was $2.0 \mathrm{~cm}(T \min =0.1 \mathrm{~cm}$; $T \max =5.0 \mathrm{~cm})$ when the distance from the nipple was less than 4.55 and $2.0 \mathrm{~cm}$ 
Table 6 Univariate analysis of MRI findings for predicting positive resection margin

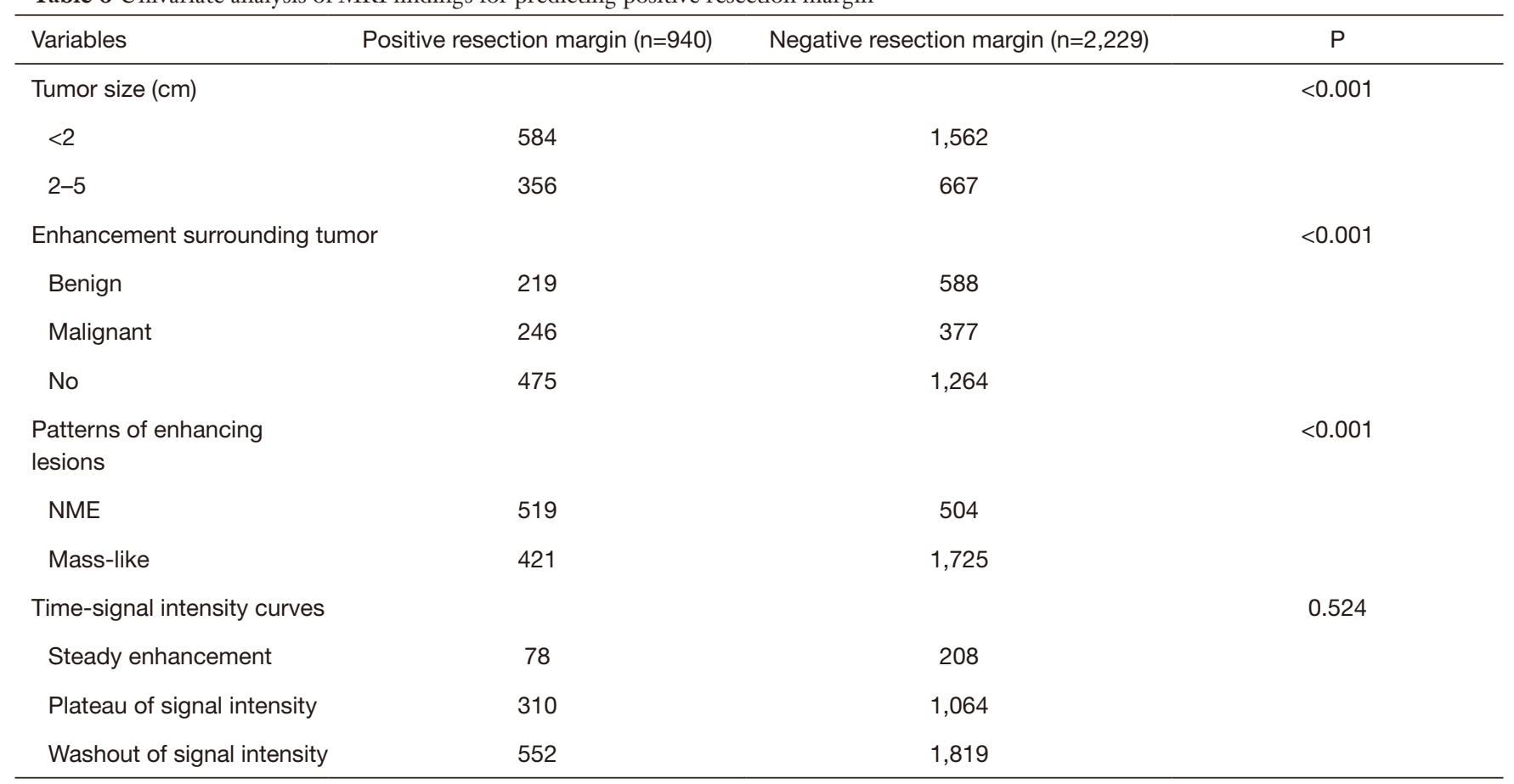

MRI, magnetic resonance imaging; NME, non-mass enhancement.

Table 7 Multivariate analysis of MRI findings for predicting positive resection margin

\begin{tabular}{lrcc}
\hline Variables & OR & $95 \% \mathrm{Cl}$ & $\mathrm{P}$ \\
\hline Tumor size $(\mathrm{cm})$ & & & \\
$<2$ & 0.706 & $0.596-0.836$ & $<0.001$ \\
$2-5$ & Ref & & \\
Enhancement surrounding tumor & & & \\
Benign & 1.051 & $0.863-1.279$ & 0.622 \\
Malignant & 1.647 & $1.302-2.083$ & $<0.001$ \\
No & Ref & & \\
Patterns of enhancing lesions & & & \\
NME & 4.433 & $3.645-5.393$ & 0.001 \\
Mass & Ref & & \\
\hline
\end{tabular}

$\mathrm{MRI}$, magnetic resonance imaging; OR, odds ratio; $\mathrm{Cl}$, confidence interval; NME, non-mass enhancement.

(Tmin $=0.7 \mathrm{~cm}$; Tmax $=4.5 \mathrm{~cm}$ ) when the distance from the nipple was more than $4.55 \mathrm{~cm}$. As there was no significant difference in tumor size between the two groups $(\mathrm{P}=0.441)$, $4.55 \mathrm{~cm}$ from the nipple can be regarded as the optimal diagnostic cut-off point.

\section{MRI and positive section margin}

Analysis of associations between MRI Findings and a positive resection margin was conducted. Univariate analysis showed that a FSA initial positive resection margin significantly correlated with malignant enhancement of the surrounding tumor $(\mathrm{P}<0.001)$, larger tumor size $(\mathrm{P}<0.001)$, and patterns of enhancing lesions [non-mass enhancement (NME); $\mathrm{P}<0.001$ ] (Table 6), and there was no statistically significant difference in the time-signal intensity curves ( $>0.05$; Table 6). Logistic analysis showed that malignant enhancement of the surrounding tumor $(\mathrm{OR}=1.647$; $\mathrm{P}<0.001)$, tumor size $(\mathrm{OR}=0.706 ; \mathrm{P}<0.001)$, and the patterns of enhancing lesions ( $\mathrm{NME}$; $\mathrm{OR}=4.433 ; \mathrm{P}<0.001$ ) were all independent predictors for a positive section margin (Table 7).

\section{Survival analysis}

From 2005 to 2013, the annual number of BCS conducted increased significantly, from 219 cases in 2005 to 771 cases in 2015 (Figure 2A). The percentages of BCS in those breast cancer cases were $11.4 \%, 12.3 \%, 12.7 \%, 12.8 \%$, 
A

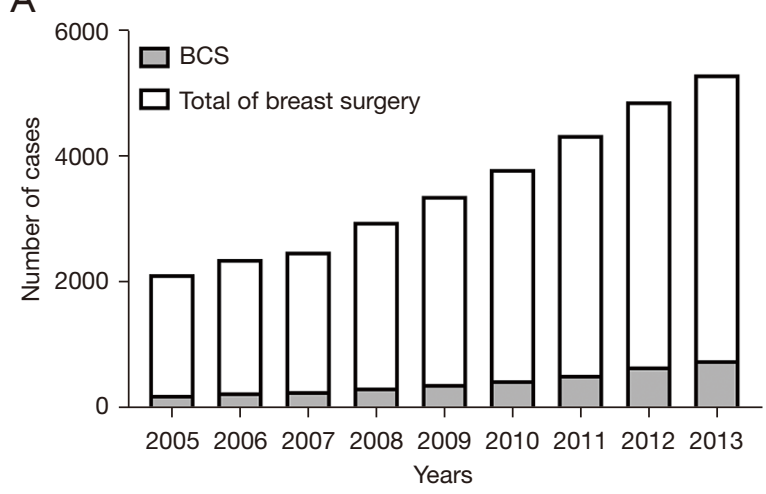

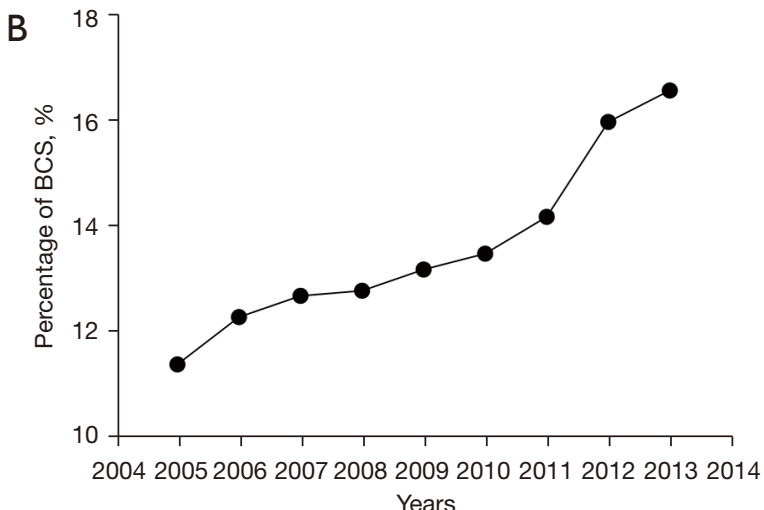

Figure 2 Breast cancer incidence and BCS prevalence. (A) Annual case number of breast cancer surgery and BCS treated in the hospital; (B) percentage of BCS cases in annual breast cancer surgery cases. BCS, breast conserving surgery.

$13.2 \%, 13.5 \%, 14.2 \%, 16.1 \%$, and $16.6 \%$ in years 2005 , 2006, 2007, 2008, 2009, 2010, 2011, 2012 and 2013, respectively (Figure $2 B$ ).

We analyzed the survival trend of breast cancer patients after increasing the proportion of BCS by comparing the LRRFS, DMFS, and OS of patients who underwent BCT between 2005 to 2010 and 2011 to 2013. The 5-year LRRFS of 2005-2010 and 2011-2013 were 97.2\% and 98.4\%, respectively, with significant difference $(\mathrm{P}=0.030$; Figure $3 A)$, and the 5-year DMFS of 2005-2010 and 2011-2013 were $94.1 \%$ and $95.2 \%$, respectively, without significant difference $(\mathrm{P}=0.110$; Figure $3 B)$. The OS of 2005-2010 and $2011-2013$ were $96.4 \%$ and $97.9 \%$ respectively, with significant difference $(\mathrm{P}=0.011$; Figure $3 C)$.

\section{Discussion}

Our study shows that socioeconomic factors largely effects the choice of BCS in China. Breast cancer patients with higher income and medical insurance are more likely to choose BCS, which is consistent with the results of similar studies in Chinese populations elsewhere (12,13). This might be due to patients having to undergo 20-25 days of radiation therapy after BCS, which increases the cost of treatment by an additional 10,000-15,000 RMB. The fear of the side effects of radiotherapy also affects the implementation of BCS. There is a lack of correct understanding of the nature of the disease, radiotherapy methods and curative effects.

In addition to controlling the influence of income level, compared with patients who lived in rural areas, breast cancer patients who lived in urban areas were more likely to choose BCS, which may indicate an uneven distribution of medical resources, such as the scarcity of radiotherapy equipment in rural hospitals, or a hesitancy of rural patients to leave their communities to obtain care in urban areas. We also found that patients with a higher education level were more willing to choose BCS, some patients are more worried about recurrence after treatment. The psychological pressure of breast preservation treatment may affect their quality of life more than mastectomy. When answering the question "is the effect of breast conserving treatment the same as that of mastectomy", doctors' vague answer is often one of the reasons why patients refuse breast conserving treatment. The guiding role of medical practitioners: knowledge level, aesthetic concept and current doctorpatient relationship are also one of the limiting factors for BCT. The patients with a higher education level can better understand the significance of BCS and have higher aesthetic requirements, which may be related to their ability to understand its safety and communicate with doctors more effectively.

At the same time, we also found that compared with minority populations, Han breast cancer patients were more willing to choose BCS. During the investigation, we found that some minorities had their own unique ethnic customs and beliefs, which makes them not want to retain the diseased breast. However, due to the large number of ethnic minorities in China and the fact that some live in specific areas, we did not enroll enough minority breast cancer patients, which limited our further exploration of their views.

Compared with mastectomy, the most important disadvantage of BCS is the higher risk of local recurrence 

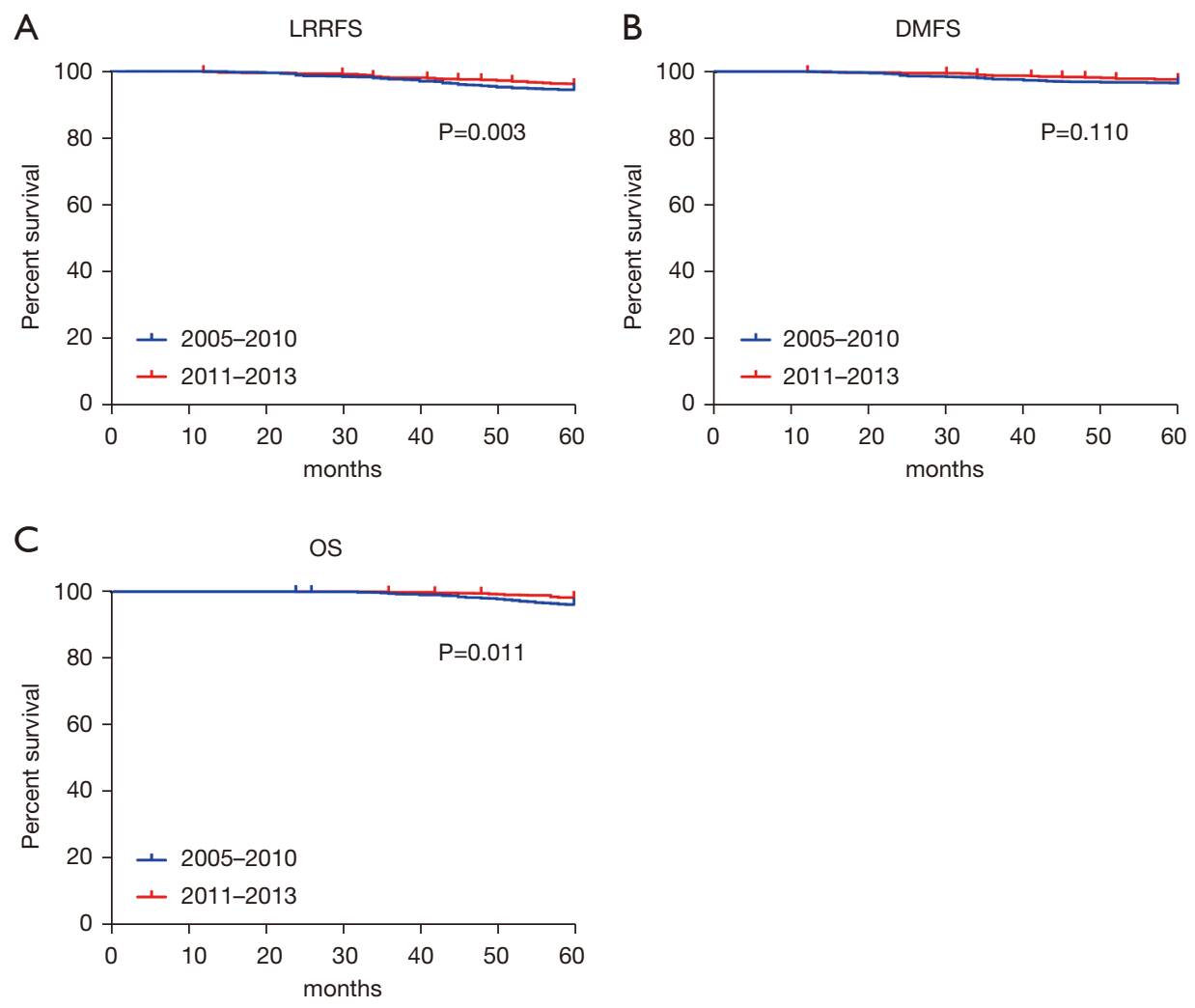

Figure 3 Survival analysis of breast cancer patients who underwent BCS between 2005 to 2010 and 2011 to 2013. (A) LRRFS; (B) DMFS; (C) OS. BCS, breast conserving surgery; LRRFS, locoregional recurrence-free survival; DMFS, distant metastasis-free survival; OS, overall survival.

due to the presence of residual small tumors at the surgical margin (14). Negative surgical margins are essential to reduce local recurrence, so the most important part in BCS is to achieve a negative surgical margin under the microscope. In BCS, the rate of reoperation can be greatly reduced by the use of FSA to evaluate the margin state during surgery $(15,16)$. Therefore, this study aimed at analyzing the clinicopathological factors and MRI findings affecting the positive FSA resection margin.

Several studies have suggested the predictors of positive surgical margins include lobular histology, LVI, insufficient preoperative diagnosis, larger tumor size, younger age, presence of ductal carcinoma in situ (DCIS), and the detection of microcalcification $(17,18)$. In our study, we found that the closer the tumor was to the nipple, the greater the possibility of a positive section margin $(\mathrm{P}=0.009)$. The ROC curve analysis showed that the distance from the nipple was $4.55 \mathrm{~cm}$ as the optimal cut-off value, and there was no significant relationship with tumor size $(\mathrm{P}=0.411)$.
Therefore, the distance from the nipple was further determined as an independent factor affecting the positive section margin of BCS, but its predictive value and accuracy still need to be further determined by multi-center studies and larger sample sizes.

This study showed that non-infiltrating cancer was riskier than infiltrating non-specific cancer in BCS. Noninfiltrating cancer breast cancer, also known as carcinoma in situ, includes lobular carcinoma in situ and intraductal carcinoma. In addition, multiple studies have demonstrated that DCIS components are predictive factors for residual lesions (17-19). DCIS can spread along the ducts and tends to exceed the range defined by negative resection margins. Therefore, a sufficiently large section margin is required to reduce recurrence.

Our study showed that preoperative MRI can reduce the positive rate of surgical resection margin in BCS, although its value is controversial. In a preoperative evaluation of 123 breast cancer cases using MRI, Obdeijn et al. (20) 
showed that compared with ultrasound and mammography, preoperative MRI examination could significantly reduce the positive rate of surgical margin in BCS, from $29.3 \%$ to $15.8 \%$. However, Shin et al. (21) showed that the positive rate of surgical margin was $23.4 \%$ in the preoperative MRI group and $23 \%$ in the preoperative non-MRI group, without statistically significant difference $(\mathrm{P}>0.05)$. In this study, we analyzed the association between MRI findings with positive section margin, and the results showed that malignant enhancement surrounding the tumor is an independent predictor of positive section margin. Although dynamic enhanced MRI scanning can well display abnormal changes around the tumor, including benign enhancing signs (background reinforcement or adenosis) or malignant signs (such as composition of DCIS), using MRI findings alone it is difficult to identify the nature of enhancement. According to Kim et al. (22), the NME group had the highest resection rate after BCS $(22.2 \% ; \mathrm{P}=0.02)$, and Kang et al. (23) reported NME was significantly associated with a positive resection margin. Similar to previous studies, NME was an independent predictor of a positive resection margin compared with mass without $\mathrm{NME}(\mathrm{OR}=4.433$; $\mathrm{P}<0.001$ ), which might be explained by the presence of a DCIS component. DCIS more commonly presented as NME than a mass on MRI. Therefore, we should pay attention to the risk of NME and expand the section margin during surgery. When surgeons perform BCS, the design of breast conserving margin should be combined with clinical and imaging features to further reduce the proportion of positive margin, so as to improve the success rate of breast conserving. The implementation of breast-conserving surgery should be carried out in accordance with the above points.

In conclusion, socioeconomic factors in China largely influence the choice of surgical procedures for breast cancer patients. Public education around BCS should increase to reduce the impact of socioeconomic factors on the choice of BCS, the establishment of multidisciplinary diagnosis and treatment mode in Department of breast surgery, imaging, pathology and radiotherapy can enable patients to have a comprehensive understanding of the treatment before starting treatment. This understanding can further affect patients' choice of breast cancer surgery. Hospitals should hold patient churches regularly to exchange professional knowledge between doctors and patients and between patients, which can not only strengthen the sense of trust between doctors and patients, but also enable patients to understand diseases more correctly and make more suitable medical decisions. And preoperative MRI should be encouraged in patients preparing for this surgery. Clinicopathological characteristics (distance from the nipple, T stage, pathological subtype, and LVI) and MRI findings (tumor size, enhancement surrounding tumor, and NME) are significantly associated with a positive resection margin in breast cancer patients.

\section{Acknowledgments}

Funding: This study was supported by research funding from the Tianjin Municipal Science and Technology Committee (No. 19YFZCSY00030), and General Project of Scientific Research Program of Tianjin Municipal Education Commission (No. 2019KJ186).

\section{Footnote}

Reporting Checklist: The authors have completed the STROBE reporting checklist. Available at https:// gs.amegroups.com/article/view/10.21037/gs-22-25/rc

Data Sharing Statement: Available at https://gs.amegroups. com/article/view/10.21037/gs-22-25/dss

Conflicts of Interest: All authors have completed the ICMJE uniform disclosure form (available at https://gs.amegroups. com/article/view/10.21037/gs-22-25/coif). The authors have no conflicts of interest to declare.

Ethical Statement: The authors are accountable for all aspects of the work in ensuring that questions related to the accuracy or integrity of any part of the work are appropriately investigated and resolved. All procedures performed in this study were in accordance with the Declaration of Helsinki (as revised in 2013). The study was approved by the Ethics Committee of Tianjin Medical University Cancer Institute and Hospital (No. bc2015014) and the need for individual consent was waived due to the retrospective nature of the present study.

Open Access Statement: This is an Open Access article distributed in accordance with the Creative Commons Attribution-NonCommercial-NoDerivs 4.0 International License (CC BY-NC-ND 4.0), which permits the noncommercial replication and distribution of the article with the strict proviso that no changes or edits are made and the original work is properly cited (including links to both the 
formal publication through the relevant DOI and the license). See: https://creativecommons.org/licenses/by-nc-nd/4.0/.

\section{References}

1. Fisher B, Anderson S, Bryant J, et al. Twenty-year followup of a randomized trial comparing total mastectomy, lumpectomy, and lumpectomy plus irradiation for the treatment of invasive breast cancer. $\mathrm{N}$ Engl J Med 2002;347:1233-41.

2. van Dongen JA, Voogd AC, Fentiman IS, et al. Long-term results of a randomized trial comparing breast-conserving therapy with mastectomy: European Organization for Research and Treatment of Cancer 10801 trial. J Natl Cancer Inst 2000;92:1143-50.

3. Jacobs L. Positive margins: the challenge continues for breast surgeons. Ann Surg Oncol 2008;15:1271-2.

4. Habermann EB, Abbott A, Parsons HM, et al. Are mastectomy rates really increasing in the United States? J Clin Oncol 2010;28:3437-41.

5. Biganzoli L, Marotti L, Hart CD, et al. Quality indicators in breast cancer care: An update from the EUSOMA working group. Eur J Cancer 2017;86:59-81.

6. Fan L, Strasser-Weippl K, Li JJ, et al. Breast cancer in China. Lancet Oncol 2014;15:e279-89.

7. Liu JJ, Zhang S, Hao X, et al. Breast-conserving therapy versus modified radical mastectomy: socioeconomic status determines who receives what--results from case-control study in Tianjin, China. Cancer Epidemiol 2012;36:89-93.

8. Singletary SE. Surgical margins in patients with early-stage breast cancer treated with breast conservation therapy. Am J Surg 2002;184:383-93.

9. Wazer DE, DiPetrillo T, Schmidt-Ullrich R, et al. Factors influencing cosmetic outcome and complication risk after conservative surgery and radiotherapy for early-stage breast carcinoma. J Clin Oncol 1992;10:356-63.

10. Chung A, Saouaf R, Scharre K, et al. The impact of MRI on the treatment of DCIS. Am Surg 2005;71:705-10.

11. Del Frate C, Borghese L, Cedolini C, et al. Role of presurgical breast MRI in the management of invasive breast carcinoma. Breast 2007;16:469-81.

12. Suen D, Chow L, Kwong A. Breast-conserving surgery in Hong Kong Chinese women. World J Surg 2008;32:2549-53.

13. Prehn AW, Topol B, Stewart S, et al. Differences in treatment patterns for localized breast carcinoma among Asian/Pacific islander women. Cancer 2002;95:2268-75.

14. Leong C, Boyages J, Jayasinghe UW, et al. Effect of margins on ipsilateral breast tumor recurrence after breast conservation therapy for lymph node-negative breast carcinoma. Cancer 2004;100:1823-32.

15. Camp ER, McAuliffe PF, Gilroy JS, et al. Minimizing local recurrence after breast conserving therapy using intraoperative shaved margins to determine pathologic tumor clearance. J Am Coll Surg 2005;201:855-61.

16. Weinberg E, Cox C, Dupont E, et al. Local recurrence in lumpectomy patients after imprint cytology margin evaluation. Am J Surg 2004;188:349-54.

17. Kurniawan ED, Wong MH, Windle I, et al. Predictors of surgical margin status in breast-conserving surgery within a breast screening program. Ann Surg Oncol 2008;15:2542-9.

18. Aziz D, Rawlinson E, Narod SA, et al. The role of reexcision for positive margins in optimizing local disease control after breast-conserving surgery for cancer. Breast J 2006;12:331-7.

19. Darvishian F, Hajdu SI, DeRisi DC. Significance of linear extent of breast carcinoma at surgical margin. Ann Surg Oncol 2003; 10:48-51.

20. Obdeijn IM, Tilanus-Linthorst MM, Spronk S, et al. Preoperative breast MRI can reduce the rate of tumorpositive resection margins and reoperations in patients undergoing breast-conserving surgery. AJR Am J Roentgenol 2013;200:304-10.

21. Shin HC, Han W, Moon HG, et al. Limited value and utility of breast MRI in patients undergoing breast-conserving cancer surgery. Ann Surg Oncol 2012;19:2572-9.

22. Kim OH, Kim SJ, Lee JS. Enhancing patterns of breast cancer on preoperative dynamic contrast-enhanced magnetic resonance imaging and resection margin in breast conserving therapy. Breast Dis 2016;36:27-35.

23. Kang JH, Youk JH, Kim JA, et al. Identification of Preoperative Magnetic Resonance Imaging Features Associated with Positive Resection Margins in Breast Cancer: A Retrospective Study. Korean J Radiol 2018;19:897-904.

(English Language Editor: B. Draper)

Cite this article as: Liu J, Zhou D, Pan T, Cao L, Yang Q, Zhang J. Socioeconomic and clinical factors affecting the proportion of breast conserving surgery in Chinese women with breast cancer. Gland Surg 2022;11(2):341-351. doi: 10.21037/ gs-22-25 